\title{
Détermination de la bio-origine des sulfures de fer présents dans les systèmes de corrosion anoxiques par analyse de la composition isotopique du soufre en nanoSIMS
}

\author{
Sophie Grousset ${ }^{1,2,3, a}$, Smaïl Mostefaoui ${ }^{4}$, Camille Chautard ${ }^{5}$, Alexandre Dauzeres ${ }^{2}$, \\ Didier Crusset $^{3}$, Valérie Deydier ${ }^{3}$, Yannick Linard ${ }^{3}$, Philippe Dillmann ${ }^{1}$, Florence \\ Mercier-Bion ${ }^{1}$ et Delphine Neff $^{1}$
}

Reçu le 6 juin 2016, accepté le 2 décembre 2016

\begin{abstract}
Résumé - Cette étude est consacrée à la détermination de l'origine biotique ou abiotique de sulfures de fer formés au cours de processus de corrosion anoxiques par l'analyse de la composition isotopique du soufre. Usuellement ces analyses sont réalisées par IRMS (Isotopic Ratio Mass Spectrometry) sur des sulfures et des sulfates préalablement dissous. Dans cette étude, en revanche, une nouvelle approche est utilisée, basée sur des analyses isotopiques locales de surface réalisées en nanoSIMS afin de conserver l'information sur la localisation des produits de corrosion. Deux types d'échantillons ont été étudiés : un échantillon (CBCC) corrodé pendant 13 mois en conditions contrôlées, et un échantillon archéologique corrodé pendant environ 2000 ans dans des conditions mal connues. Lorsque le système de corrosion est parfaitement connu, tel que le cas de l'échantillon CBCC, l'analyse isotopique locale par nanoSIMS permet effectivement de conclure sur la (bio-)origine des liserés de sulfures de fer formés. En revanche, lorsque la composition isotopique de la source de sulfates est inconnue, comme dans le cas de l'échantillon archéologique, il n'est pas toujours possible de conclure sur l'origine des sulfures de fer observés.
\end{abstract}

Mots clés : Corrosion anoxique / sulfures de fer / bactéries / fractionnement isotopique du soufre / nanoSIMS

\begin{abstract}
Characterisation and determination of the bio-origin of iron sulphides in anoxic corrosion systems thanks to isotopic analyses by nanoSIMS. This study is devoted to the determination of the bacterial or inorganic origin of iron sulphides formed during anoxic corrosion processes by sulfur isotopic composition analyses. Broadly, these analyses are realized by IRMS on dissolved sulphides and sulphates. Yet in this study, surface local isotopic analyses are realized by nanoSIMS in order to preserve the information about the corrosion products' localization. Two kinds of samples are studied: a sample (CBCC) corroded during 13 months in controlled laboratory conditions; and one archeological sample corroded during about 2000 years in not very well known conditions. When the corrosion system is perfectly well known, as for the CBCC sample, the local isotopic analyses by nanoSIMS actually enable to conclude on the (bio-)origin of the iron sulphides observed. However, when the isotopic composition of the sulphate's source is unknown, as for the archeological sample, the origin of iron sulphides cannot always be determined.
\end{abstract}

Key words: Anoxic corrosion / Iron sulphide / Sulphur isotopic fractionation / NanoSIMS / Bacteria

\footnotetext{
a Auteur de correspondance : sophie.grousset@cea.fr

1 LAPA, NIMBE-IRAMAT, CEA/CNRS, Université Paris Saclay, UMR 3685 et IRAMAT UMR 5060 CNRS, CEA Saclay, 91191 Gif-sur-Yvette, France

2 IRSN, PRP-DGE/SRTG/LETIS, B.P. 17, 92262 Fontenay-aux-Roses Cedex, France

3 Andra, Direction de la recherche et du développement, 1-7 rue Jean Monnet, 92298 Châtenay-Malabry Cedex, France

4 IMPMC-COSMO, Museum National d'Histoire Naturelle, 75231 Paris Cedex 05, France

5 IRSN, PRP-DGE/SEDRAN/BERIS, 92262 Fontenay-aux-Roses Cedex, France
} 


\section{Introduction}

La formation de sulfures de fer lors de processus de corrosion anoxique constitue une problématique majeure que ce soit pour la conservation d'objets archéologiques in situ sur leur site d'enfouissement [1-3] ou pour la prévision de la durée de vie d'ouvrages métalliques enfouis dans les sols tels que les oléoducs $[4,5]$ ou encore les barrières en acier non ou faiblement allié, surconteneurs et chemisage, prévus dans le cadre du stockage des déchets nucléaires de haute activité en milieu géologique profond $[6,7]$. En effet, la formation de sulfures de fer est susceptible de modifier les processus de corrosion. Ainsi la formation d'une couche hétérogène de sulfures de fer, phases conductrices, pourrait notamment entraîner un phénomène de corrosion galvanique [8] et/ou des hétérogénéités de $\mathrm{pH}$ [9] conduisant à l'accélération des processus de corrosion. La formation d'un dépôt homogène de sulfures de fer constituerait quant à elle une couche passivante inhibant les processus de corrosion [10-12]. Afin de prévoir la durée de vie d'échantillons ferreux en conditions anoxiques, il est donc nécessaire de mieux comprendre les processus de formation conduisant à la présence de sulfures de fer parmi les produits de corrosion formés. Notamment, l'intervention de bactéries telles que les bactéries sulfato-réductrices, dans le processus de formation des sulfures de fer, est envisagée. En effet, ces bactéries sont capables de transformer les sulfates du milieu environnant en sulfures [13]. Or, lorsque la formation d'anions sulfures se produit simultanément au processus de dissolution anodique du fer, la précipitation de phases de sulfures de fer peut avoir lieu. Ainsi en milieu pauvre en sulfures, l'observation de sulfures de fer au sein des couches de produits de corrosion est usuellement associée à l'activité de bactéries sulfato- (ou thiosulfato-)réductrices. Cependant dans les milieux riches en composés sulfurés (ex : argiles riches en pyrite), la formation de sulfures de fer pourrait également se produire par le biais de mécanismes totalement inorganiques de dissolution-reprécipitation. Dès lors, comment distinguer les sulfures de fer ayant une origine bactérienne de ceux formés selon des mécanismes de corrosion purement inorganiques?

Dans la littérature, des analyses isotopiques du soufre réalisées en global sur des échantillons de solution aqueuse de laboratoire ont montré que la réduction microbienne des sulfates aboutit à des sulfures déplétés en soufre 34 jusqu'à des valeurs de fractionnement isotopique

$$
\delta^{34} S=\frac{R^{34} S(\text { sulfures })-R^{34} S(\text { sulfates })}{R^{34} S(\text { sulfates })} \times 1000
$$

avec $R^{34} S=\frac{I\left({ }^{34} S\right)}{I\left({ }^{32} S\right)}$

pouvant atteindre $\sim 70 \%$ [14-17]. $\delta^{34} S$ représente ici le fractionnement isotopique entre les sulfures formés et les sulfates résiduels en solution. En effet, les bactéries utilisent préférentiellement les sulfates les plus légers, c'està-dire ceux composés de l'isotope le plus léger du soufre $\left({ }^{32} \mathrm{~S}\right)$, par rapport aux sulfates les plus lourds contenant des isotopes plus lourds du soufre $\left({ }^{33} \mathrm{~S},{ }^{34} \mathrm{~S},{ }^{36} \mathrm{~S}\right)$ [18]. Les sulfures issus de l'activité métabolique seraient donc enrichis en soufre 32, conduisant à des fractionnements isotopiques de plusieurs dizaines de pour mille. En revanche, dans le cas de processus inorganiques, les fractionnements isotopiques attendus sont beaucoup plus faibles en valeurs absolues, de l'ordre de quelques pour mille [19-21]. L'étude de la composition isotopique du soufre au sein des sulfures de fer pourrait donc permettre la détermination de la bio-origine de ces phases.

De nombreuses études recensées dans la littérature semblent conforter la pertinence de l'étude isotopique pour la détermination de l'origine biotique ou abiotique des sulfures de fer. Ces études sont consacrées à deux types d'échantillons : des échantillons naturels c'est-àdire des minéraux de sulfures de fer et leur solution environnante prélevés directement dans le milieu naturel $[15,18,19,22]$ ou des échantillons issus d'expériences de laboratoire $[14,23,24]$. Le plus souvent les compositions isotopiques sont déterminées en IRMS (Isotopic Ratio Mass Spectrometry) après dissolution des sulfures et des sulfates. Cette méthode permet de déterminer les compositions isotopiques moyennes des sulfates et des sulfures. Ces mesures renseignent donc sur le fractionnement isotopique moyen des sulfures par rapport aux sulfates. D'après l'hypothèse exposée précédemment selon laquelle les sulfures de fer d'origine bactérienne présenteraient un fractionnement isotopique pouvant aller jusqu'à plusieurs dizaines de pour mille, il serait alors possible de conclure sur la bio-origine des sulfures de fer.

L'étude décrite ci-après est consacrée à deux échantillons : un premier échantillon corrodé en laboratoire sur une durée moyenne (13 mois) et pour lequel les conditions de corrosion sont bien connues; et un échantillon archéologique corrodé pendant près de deux mille ans dans des conditions de corrosion mal connues. L'objectif est de déterminer l'origine biotique ou abiotique des sulfures de fer formés dans ces deux types d'échantillons tout en préservant l'information sur la localisation des phases de sulfures de fer au sein de la couche de produits de corrosion. Afin de préserver la morphologie de la couche de produits de corrosion, les compositions isotopiques des sulfures de fer sont déterminées localement par nanoSIMS sans nécessiter de dissolution préalable des sulfures. Nous avons ensuite étudié la faisabilité de la détermination de l'origine des sulfures de fer par analyses isotopiques en fonction des informations accessibles sur le système de corrosion.

\section{Matériels et méthode}

\subsection{Références}

Deux échantillons standards ont été utilisés pour la calibration des instruments d'analyse isotopique : la Troilite Canon Diablo $\left({ }^{34} \mathrm{~S} /{ }^{32} \mathrm{~S}(\mathrm{CDT})=0,0450045\right)[19]$ dans le cas du nanoSIMS; la Troilite Vienna-Canon Diablo 


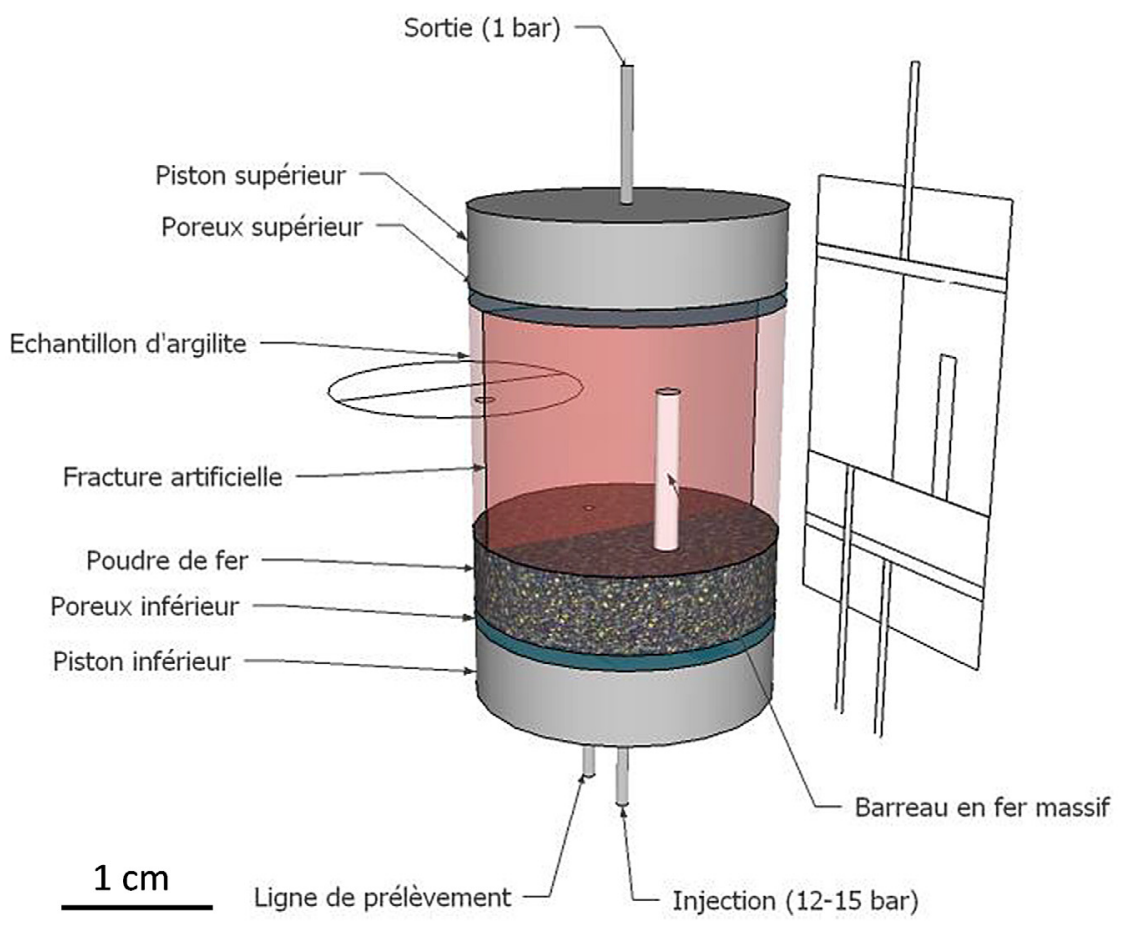

Fig. 1. Schéma de la cellule de percolation biotique.

$\left({ }^{34} \mathrm{~S} /{ }^{32} \mathrm{~S}(\mathrm{~V}-\mathrm{CDT})=0,0441626\right)[25]$ dans le cas de l'EACF-IRMS.

\section{2 Échantillon de laboratoire}

L'échantillon CBCC est issu d'une expérience de corrosion anoxique en cellule de percolation biotique à $60^{\circ} \mathrm{C}$ pendant 13 mois (Fig. 1) [6,26]. Tous les éléments composant cette cellule ont été préalablement stérilisés. La cellule de percolation cylindrique est constituée par un tapis de poudre de fer métal compactée et calibrée $\left(d_{\text {particule }} \leq\right.$ $60 \mu \mathrm{m}, S_{\text {spécifique }}=0,13 \pm 0,02 \mathrm{~m}^{2} / \mathrm{g}$ ). La poudre de fer métal est surmontée d'un échantillon d'argilite du Toarcien prélevée sur le site de Tournemire, la plateforme expérimentale de l'IRSN (Institut de Radioprotection et de Sûreté Nucléaire) située dans la région AuvergneRhône-Alpes en France. La composition minéralogique de cette argilite est détaillée dans le Tableau 1. Enfin, un barreau de fer $(\mathrm{d}=3 \mathrm{~mm}, \mathrm{H}=1 \mathrm{~cm})$ a été inséré dans l'argilite. Une solution porale synthétique est injectée en continu par la base du dispositif à une pression comprise entre 12 et 15 bars. Cette solution traverse donc successivement la poudre de fer métal compactée puis l'échantillon d'argilite préalablement fissuré. La composition de la solution porale synthétique, détaillée dans le Tableau 2, a été choisie afin de s'approcher au maximum de la composition de l'eau porale en équilibre avec la roche argileuse de Tournemire $[27,28]$. En outre, elle doit être en équilibre avec l'argilite à $60^{\circ} \mathrm{C}$, température choisi pour cet essai. Enfin, elle doit contenir certains éléments nécessaires à la croissance bactérienne et notamment le phosphore, le carbone et l'azote.
Enfin, deux souches bactériennes, la sulfato-réductrice Thermodesulfovibrio hydrogeniphilus Hbr5T (DSM 18151) isolée dans une source d'eau chaude en Tunisie [29] et la ferri-réductrice Thermotoga subterranea strain SLT 1 isolée dans un réservoir pétrolier de l'est du bassin de Paris [30] ont été inoculées dans la cellule. Chaque souche bactérienne a d'abord été cultivée, en anoxie, sur un milieu de culture « riche » dont la composition est détaillée dans le Tableau 3. Puis les souches bactériennes ont été cultivées, toujours en anoxie, sur le milieu appauvri en éléments nutritifs et énergétiques plus représentatif de la composition de l'eau porale de Tournemire (Tab. 2). Dans ce milieu, les souches sont mises en cultures simultanément.

En début d'essai, la densité totale de bactéries inoculées dans la cellule de percolation atteignait $4 \times$ $10^{8}$ bactéries $/ \mathrm{mL}$. Au cours de l'essai, la population bactérienne est suivie au cours du temps lors de prélèvements liquides via la méthode de l'épifluorescence. Le fluorochrome utilisé est le SYTO ${ }^{\circledR} 9$ stain de ThermoFischer Scientific ${ }^{\circledR}$. Il a ainsi été observé un maintien de la population bactérienne dans la cellule biotique pendant toute la période de l'essai (13 mois). Néanmoins, une diminution nette de la population est observée après 200 jours de suivi jusqu'en fin d'essai. Cela semble avoir entraîné une diminution de l'activité bactérienne dans le système. En effet, l'activité bactérienne est estimée grâce à la consommation des sulfates. Celle-ci est élevée pendant les 100 premiers jours $(2,1 \mathrm{mmol} / \mathrm{L} / \mathrm{j})$ mais a chuté à $0,5 \mathrm{mmol} / \mathrm{L} / \mathrm{j}$ en fin d'essai. Au sein de la cellule de percolation, la nature probable des donneurs d'électrons sont le dihydrogène produit par la corrosion de la poudre 
Tableau 1. Minéralogie de l'argilite de Tournemire [27].

\begin{tabular}{cccccccc}
\hline Minéral & Kaolinite & Illite & Illite/Smectite & Chlorite & Carbonates & Quartz & Pyrite \\
\hline Proportion en masse (\%) & 10 à 20 & 5 à 15 & 20 à 30 & 1 à 5 & 10 à 40 & 10 à 20 & 2 à 5 \\
\hline
\end{tabular}

Tableau 2. Composition de la solution porale synthétique.

\begin{tabular}{cccccccc}
\hline Sel & $\left(\mathrm{NH}_{4}\right)_{2} \mathrm{SO}_{4}$ & $\mathrm{KH}_{2} \mathrm{PO}_{4}$ & $\mathrm{NaHCO}_{3}$ & $\mathrm{Na}_{2} \mathrm{SO}_{4}$ & $\mathrm{NaCl}$ & $\mathrm{KCl}$ & $\mathrm{MgCl}_{2}, 6 \mathrm{H}_{2} \mathrm{O}$ \\
\hline Concentration massique $(\mathrm{g} / \mathrm{L})$ & 0,395 & 0,041 & 0,303 & 1,155 & 0,081 & 0,054 & 0,161 \\
\hline
\end{tabular}

de fer et du fer massif, ainsi que la matière organique dissoute, notamment l'acétate, le formate, le fumarate et le lactate provenant de la roche argileuse et identifiés par C. Chautard au cours de ses travaux de thèse [26]. Ces composés organiques pourraient également constituer une source de carbone pour les bactéries. La concentration en fumarate dans l'eau porale de l'argilite de Tournemire est comprise entre 0,9 et $3,4 \mathrm{mg} / \mathrm{L}$ [26]. Les concentrations en acétate, formate et lactate sont, quant à elles, très faibles : inférieures à $0,5 \mathrm{mg} / \mathrm{L}$ [26].

\section{3 Échantillon archéologique}

Afin de comprendre les variations de rapport isotopique sur des produits de corrosion formées sur le long terme, un clou archéologique en fer, l'échantillon T6Fl2Cl3, provenant du chaland gallo-romain d'Arles Rhône 3 (Arles, Bouches-du-Rhône, France) a également été étudié [32]. L'épave date du $1^{\text {er }}$ siècle après JC et a été découverte entre $4 \mathrm{~m}$ (la poupe) et $9 \mathrm{~m}$ (la proue) de profondeur dans le Rhône. Le clou, incrusté dans le bois du navire, s'est donc corrodé pendant près de deux mille ans en conditions anoxiques au fond du Rhône.

\subsection{Préparation des échantillons}

La préparation des échantillons $\mathrm{CBCC}$ et $\mathrm{T} 6 \mathrm{Fl} 2 \mathrm{Cl} 3$ a été réalisée dans une boîte à gant anoxique en $\mathrm{PVC}$ avec système de régulation en surpression par rapport à la pression atmosphérique (Les Ateliers de Technochimie ${ }^{\circledR}$ ). L'atmosphère au sein de la boîte à gant est composée de $\mathrm{N}_{2}$ pour préserver les sulfures de fer qui sont des phases très réactives à l'oxygène. Afin de permettre l'analyse en coupe transverse des échantillons, ils ont tout d'abord été enrobés dans de la résine époxy (Specifix-20, Struers ${ }^{\circledR}$ ) puis découpés transversalement et polis sous éthanol à l'aide de papier SiC (320-4000). Le polissage est finalement perfectionné en utilisant une poudre diamantée de $1 \mu \mathrm{m}$.

\subsection{Techniques de caractérisations élémentaires et structurales}

La morphologie des échantillons préparés en coupe transversale a tout d'abord été étudiée en microscopie optique en champ clair. Puis, suite à leur métallisation par pulvérisation d'une couche de carbone, les échantillons ont été analysés en microscopie électronique à balayage à effet de champ (MEB FEG) couplé à un système de détection EDS (Energy Dispersive Spectrosocpy) de type SDD (Silicon Drift Detector). L'appareil utilisé est le JSM-7001F de JEOL et la tension d'accélération employée est de $15 \mathrm{kV}$. Ainsi, la distribution des éléments chimiques (Fe, O, S, Ca) au sein des couches de produit de corrosion a pu être étudiée avec une résolution latérale de $1 \mu \mathrm{m}$. Plus particulièrement, des liserés contenant à la fois du soufre et du fer ont pu être localisés au sein des couches. Pour cela, le logiciel Idfix de Fondis Electronics a été utilisé conjointement au logiciel Maxview de Fondis Electronic.

La microspectroscopie Raman a également été employée afin de préciser la structure cristalline des produits de corrosion et notamment la composition des liserés soufrés préalablement observés en MEB FEG-EDS. L'appareil utilisé est un spectromètre Renishaw ${ }^{\circledR}$ (InVia Raman Microscope) associé à un microscope optique Leica. Le laser employé ( $\mathrm{Nd}$ :YAG doublé) émet un rayonnement vert de longueur d'onde $\lambda=532 \mathrm{~nm}$. La résolution spatiale obtenue est de l'ordre de $1 \mu \mathrm{m}$ de diamètre. L'acquisition et le traitement des spectres Raman sont effectués avec le logiciel WIRE de Renishaw. Les temps d'acquisition sont compris entre 30 et $300 \mathrm{~s}$ par point. Les spectres obtenus sont ensuite comparés aux spectres recensés dans la littérature. Les positions des bandes associées à chacune des phases de sulfures de fer sont résumées dans le Tableau 4. Dans le cas de l'échantillon CBCC, les analyses $\mu$ Raman ont été réalisées en cellule anoxie afin de préserver les échantillons de l'oxygène. Le spectre associé au verre de la cellule est présenté Figure 2 et présente deux bandes larges autour de 400 et $800 \mathrm{~cm}^{-1}$ caractéristiques d'une matrice vitreuse.

\subsection{Méthode de détermination de la valeur de fractionnement isotopique du soufre}

Le fractionnement isotopique du soufre, $\delta^{34} \mathrm{~S}$, est toujours calculé par rapport à une référence (cf. Eq. (1)). On peut prendre comme référence des sulfures solides, la CDT ou V-CDT ou bien les sulfates résiduels.

Dans le cas de l'échantillon CBCC nous avons utilisé les sulfates résiduels afin d'obtenir une comparaison immédiate entre la composition isotopique des sulfures formés et celle de la source initiale de sulfates :

$$
\delta^{34} S=\frac{R^{34} S(\text { sulfures })-R^{34} S(\text { sulfates })}{R^{34} S(\text { sulfates })} \times 1000,
$$


Tableau 3. Composition des milieux préconisés par la souchothèque DSMZ de chacune des deux souches dit « milieu riche ».

\begin{tabular}{|c|c|c|}
\hline & Thermodesulfovibrio hydrogeniphilus & Thermotoga subterranea \\
\hline Tryptone (g/L) & 1,0 & \\
\hline Peptone $(\mathrm{g} / \mathrm{L})$ & & 1,0 \\
\hline Extrait de levure $(\mathrm{g} / \mathrm{L})$ & 2,0 & 0,5 \\
\hline $\mathrm{KH}_{2} \mathrm{PO}_{4}(\mathrm{~g} / \mathrm{L})$ & 0,3 & \\
\hline $\mathrm{Na}_{2} \mathrm{SO}_{4}(\mathrm{~g} / \mathrm{L})$ & 3,0 & \\
\hline $\mathrm{MgSO}_{4}, 7 \mathrm{H}_{2} \mathrm{O}$ & & 0,5 \\
\hline $\mathrm{K}_{2} \mathrm{HPO}_{4}(\mathrm{~g} / \mathrm{L})$ & 0,3 & 0,02 \\
\hline $\mathrm{NH}_{4} \mathrm{Cl}(\mathrm{g} / \mathrm{L})$ & 1,0 & 0,1 \\
\hline $\mathrm{KCl}(\mathrm{g} / \mathrm{L})$ & & 2,0 \\
\hline $\mathrm{MgCl}_{2}, 6 \mathrm{H}_{2} \mathrm{O}(\mathrm{g} / \mathrm{L})$ & 0,2 & \\
\hline $\mathrm{CaCl}_{2}, 2 \mathrm{H}_{2} \mathrm{O}$ & & 0,025 \\
\hline $\mathrm{NaCl}(\mathrm{g} / \mathrm{L})$ & 1,0 & 0,5 \\
\hline Acétate de sodium $(\mathrm{g} / \mathrm{L})$ & 0,16 & \\
\hline Cystéine - $\mathrm{HCl}(\mathrm{g} / \mathrm{L})$ & 0,5 & \\
\hline $\mathrm{NaCO}_{3}$ à $0,2 \mathrm{~mol} / \mathrm{L}(\mathrm{mL})$ & 1,0 & 1,0 \\
\hline $\mathrm{Na}_{2} \mathrm{~S}$ à $0,2 \mathrm{~mol} / \mathrm{L}(\mathrm{mL})$ & - & 2,0 \\
\hline Solution d'éléments traces $(\mathrm{mL})$ & $\begin{array}{l}\text { 1,0 (solution d'éléments } \\
\text { traces du milieu M1 [31]) }\end{array}$ & $\begin{array}{c}10,0 \text { (solution d'éléments } \\
\text { traces du milieu } 141 \text { de la DSMZ) }\end{array}$ \\
\hline $\begin{array}{l}\text { Mélange de vitamines détaillé } \\
\text { dans la fiche DSMZ du milieu } 141(\mathrm{~mL})\end{array}$ & 1,0 & 1,0 \\
\hline
\end{tabular}

Tableau 4. Positions des pics principaux des spectres Raman associées aux composés soufrés reportés dans la littérature. Les nombres d'onde reportés en gras correspondent à la position du (des) pic(s) le(s) plus intense(s) associé(s) à chaque phase de sulfure de fer considérée.

\begin{tabular}{|c|c|c|}
\hline Sulfure de fer & $\begin{array}{c}\text { Principales bandes de vibration } \\
\text { Raman à partir de } 200 \mathrm{~cm}^{-1}\left(\mathrm{~cm}^{-1}\right)\end{array}$ & Références \\
\hline Mackinawite nanocristalline FeS & 208 et 282 & $\begin{array}{l}\text { Boughriet et al., } 1997[33] \\
\text { Bourdoiseau et al., } 2008[34] \\
\text { Bourdoiseau et al., } 2011[35]\end{array}$ \\
\hline Mackinawite cristalline (FeS) & 208,256 et 298 & $\begin{array}{l}\text { Bourdoiseau et al., } 2008 \text { [34] } \\
\text { Bourdoiseau et al., } 2011 \text { [35] }\end{array}$ \\
\hline $\begin{array}{l}\text { Mackinawite partiellement oxydée } \\
\mathrm{Fe}_{1-3 \mathrm{x}}^{\mathrm{II}} \cdot \mathrm{Fe}_{2 \mathrm{x}}^{\mathrm{III}} \cdot \mathrm{S} \text { avec } x=0,09\end{array}$ & 256,312 et 322 & Bourdoiseau et al., 2011 [35] \\
\hline Soufre élémentaire $\alpha-\mathrm{S}_{8}$ & $\begin{array}{l}\text { Pics intenses \& fins : } 223 \text { et } 474 \\
\text { Pics secondaires : } 249 \text { et } 437\end{array}$ & $\begin{array}{l}\text { Bourdoiseau et al., } 2008 \text { [34] } \\
\text { Bourdoiseau et al., } 2011 \text { [35] }\end{array}$ \\
\hline Greigite $\left(\mathrm{Fe}_{3} \mathrm{~S}_{4}\right) \mathrm{Fe}^{\mathrm{II}} \mathrm{Fe}_{2}^{\mathrm{III}} \mathrm{S}_{4}$ & $\begin{array}{c}\text { Pic intense \& large : } 350-370 \text { (parfois deux } \\
\text { bandes distinctes à } 351 \mathrm{~cm}^{-1} \text { et } 366 \mathrm{~cm}^{-1} \text { ) } \\
\text { Pic secondaire : } 250\end{array}$ & Bourdoiseau et al., 2011. [35] \\
\hline Pyrite $\left(\mathrm{FeS}_{2}\right)$ & $\begin{array}{l}\text { Pics principaux : } 343 \text { et } 379 \\
\text { Pics secondaires : } 430\end{array}$ & White, 2009 [36] \\
\hline Marcasite $\left(\mathrm{FeS}_{2}\right)$ & $\begin{array}{c}\text { Pic principal : } 323 \\
\text { Pic secondaire }: 386\end{array}$ & White, 2009 [36] \\
\hline
\end{tabular}

Les poudres de sulfates commerciales utilisées pour la réalisation de la solution synthétique injectée dans la cellule de percolation de cet échantillon ont donc été préalablement analysées en EA-CF-IRMS (Elementary Analyser Continuous Flow Isotope Ratio Mass Spectrometry). La calibration a été réalisée à l'aide du standard isotopique international VCDT.

Par contre, dans le cas de l'échantillon archéologique $\mathrm{T} 6 \mathrm{Fl} 2 \mathrm{Cl} 3$ la source initiale de sulfates à proximité du clou n'étant pas accessible, le fractionnement isotopique a été calculé par rapport au standard international CDT :

$$
\delta^{34} S=\frac{R^{34} S(\text { sulfures })-R^{34} S(C D T)}{R^{34} S(C D T)} \times 1000
$$

La composition isotopique en soufre des liserés de sulfures de fer observés a été déterminée en nanoSIMS. L'instrument utilisé à cette fin est le NanoSIMS NS0 de CAMECA ${ }^{\circledR}$ du Muséum National d'Histoire Naturelle de Paris (MNHN). Le traitement des images est réalisé avec le logiciel PV-WAVE. Lors de l'analyse, une surface de $12 \mu \mathrm{m} \times 12 \mu \mathrm{m}$ est tout d'abord abrasée par un courant d'ions primaires $\mathrm{Cs}^{+}$de $18 \mathrm{pA}$ pendant $25 \mathrm{~min}$. Puis le courant de ce faisceau d'ions est diminué pour l'acquisition de sorte qu'il atteigne une valeur d'environ $1 \mathrm{pA}$ au niveau de la surface. La surface analysée est réduite à une zone de $9 \mu \mathrm{m} \times 9 \mu \mathrm{m}$ et la résolution est fixée à 256 pixels $\times 256$ pixels (soit $35 \times 35 \mathrm{~nm}^{2} /$ pixel). L'acquisition dure 50 à 100 cycles en fonction des analyses. 


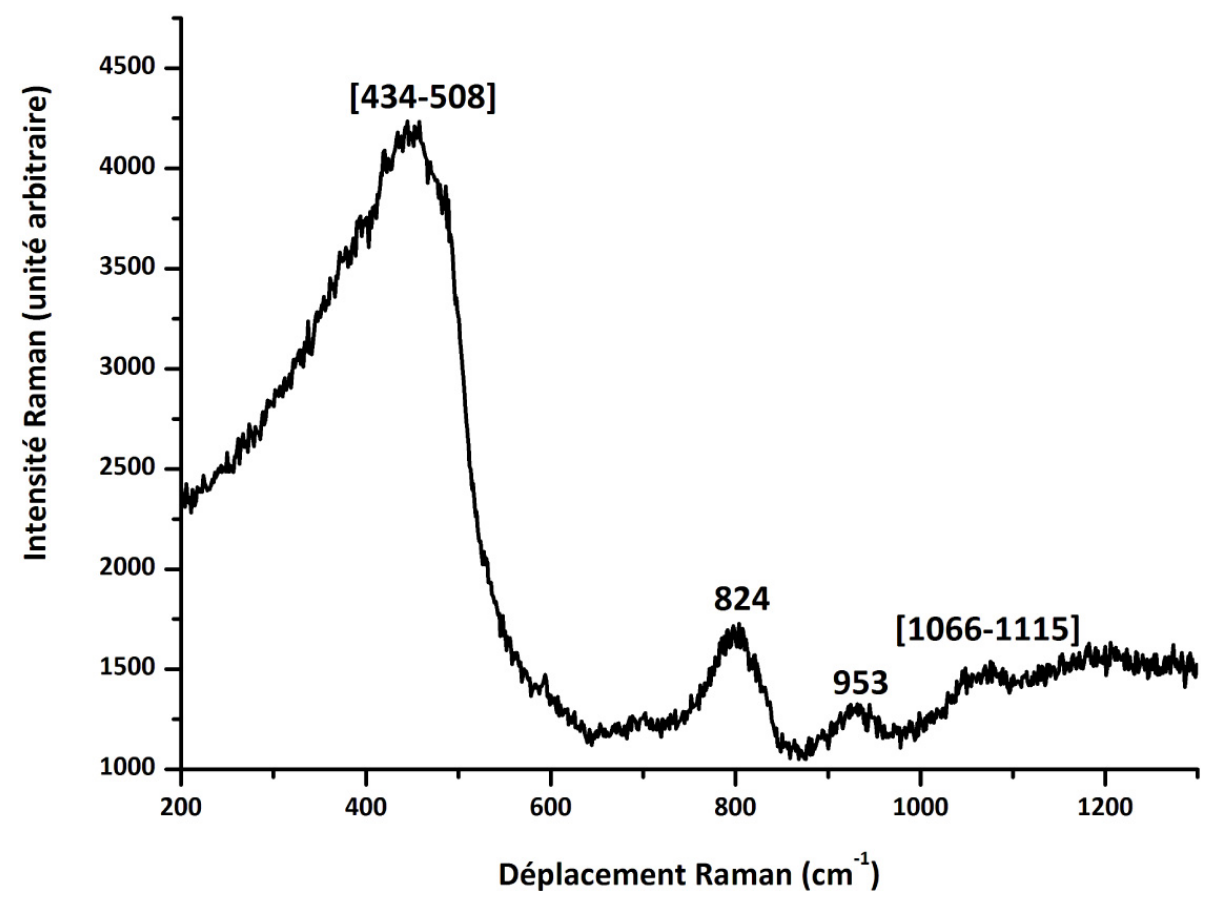

Fig. 2. Spectre Raman du verre de la cellule sous anoxie.

Plusieurs sources d'erreur peuvent impacter la valeur de la composition isotopique obtenue en nanoSIMS par rapport à la composition isotopique réelle de l'échantillon : l'effet de l'arrivée quasi-simultanée des ions secondaires sur le détecteur (《effet QSA»); et le fractionnement instrumental se produisant lors du trajet des ions secondaires entre la surface de l'échantillon et le détecteur. Finalement, chaque point des graphes présentés dans cet article correspond à une unique analyse. Les barres d'erreurs associées matérialisent l'erreur finale réalisée sur le fractionnement isotopique corrigé à la fois de l'effet QSA et du fractionnement instrumental, $\delta^{34} \mathrm{~S}$ (corr).

\section{Résultat}

\section{1 Échantillons de laboratoire}

La Figure 3 est une micrographie optique de l'échantillon CBCC réalisée en coupe transverse. Nous distinguons un liseré doré d'une dizaine de micromètres d'épaisseur à l'interface entre le barreau de fer et l'argilite. Les analyses en MEB FEG-EDS ont permis de préciser la composition élémentaire de ce liseré doré, riche en fer et soufre mais pauvre en oxygène (Fig. 4). Des mélanges à l'échelle submicrométrique de sulfures de fer de type mackinawite (nanocristalline, cristalline ou partiellement oxydée en Fe(III)), greigite, pyrite et soufre $\alpha$-S8 ont été identifiés en microspectroscopie Raman. La Figure 5 présente trois spectres représentatifs des spectres de mélanges de phases obtenus sur cette zone. Enfin, les analyses isotopiques réalisées sur ce liseré de sulfure de fer et sur les sulfates de la solution synthétique mettent

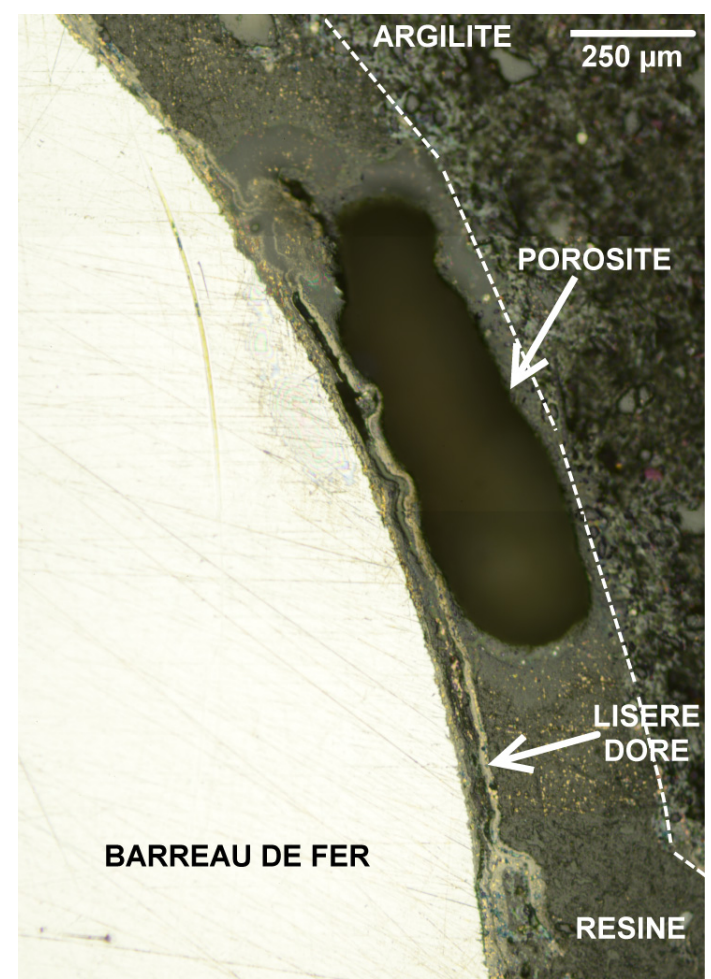

Fig. 3. Microscopie optique d'une section transverse de l'échantillon CBCC.

en évidence une déplétion en soufre 34 des sulfures de fer par rapport aux sulfates de la solution synthétique. Les fractionnements isotopiques obtenus valent environ $-4,5 \pm 5 \%$ environ quelle que soit la zone (1, 2 ou 3 de la Fig. 4c) du liseré analysée (Fig. 6). 


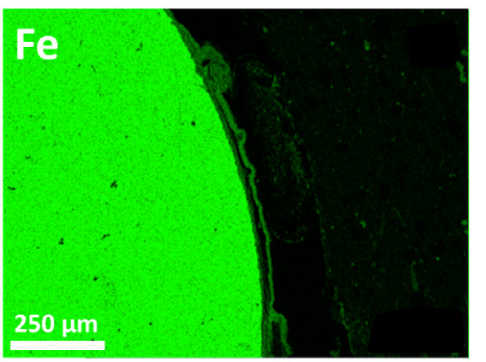

(a)

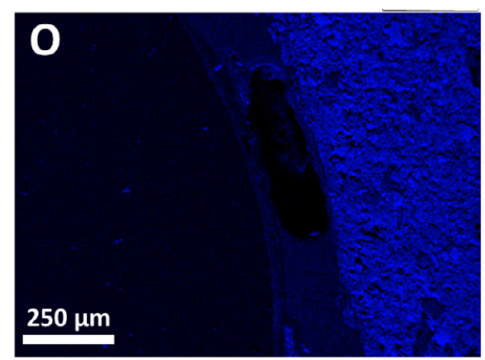

(b)

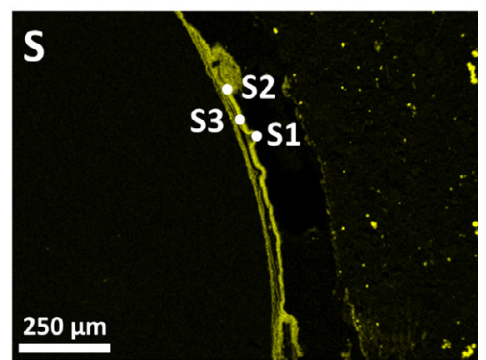

(c)

Fig. 4. Image EDS en superposition du fer (a), de l'oxygène (b) et du soufre (jaune), section transverse de l'échantillon CBCC. Les zones 1,2 et 3 correspondent respectivement aux spectres $\mu$ Raman de la Figures $5 \mathrm{a}, 5 \mathrm{~b}$ et $5 \mathrm{c}$.

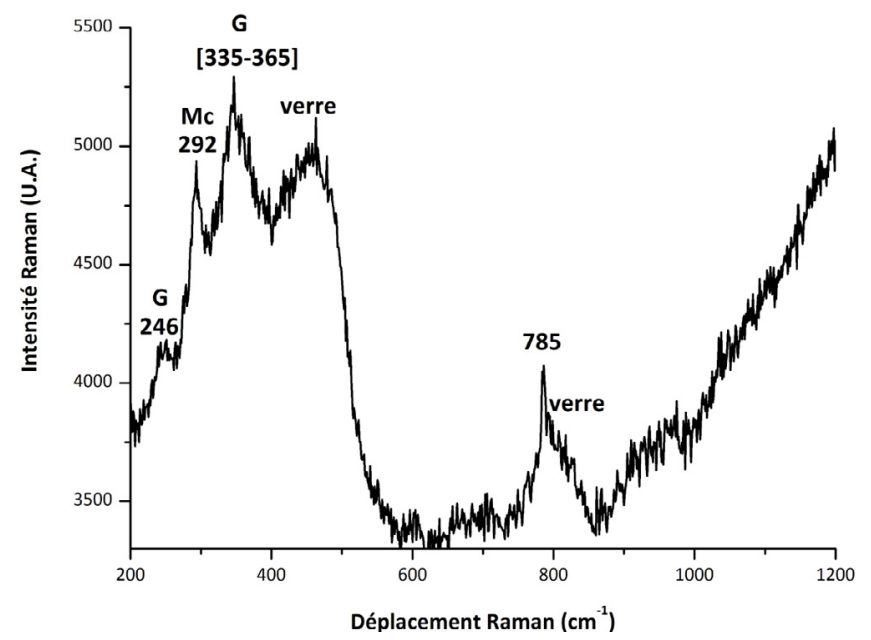

(a)

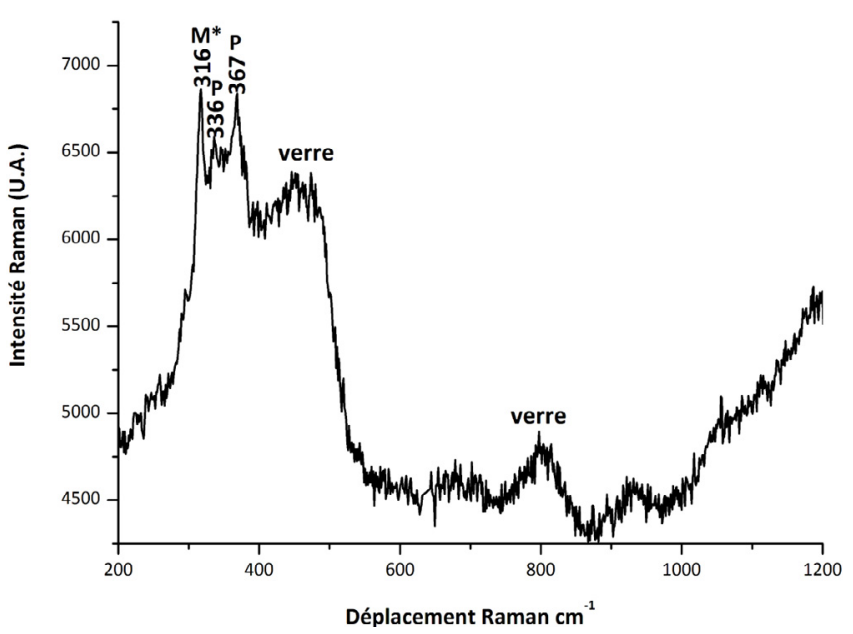

(b)

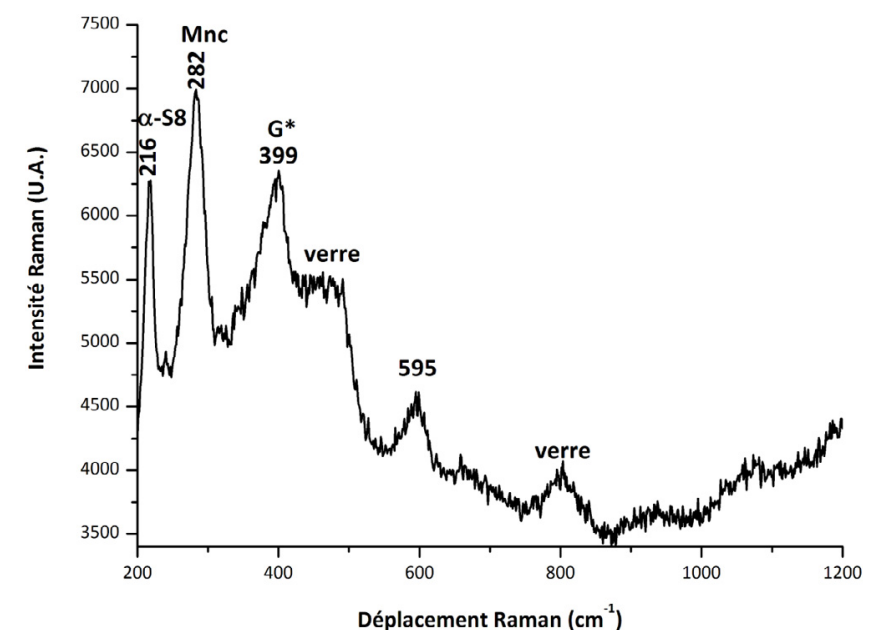

(c)

Fig. 5. Spectres $\mu$ Raman : (a) mélange greigite $(\mathrm{G})$ et mackinawite cristalline $(\mathrm{Mc})$; (b) mackinawite nanocristalline (Mnc) mélangée au soufre $\alpha$-S8 et à la goethite $\left(\mathrm{G}^{*}\right) ;(\mathrm{c})$ mélange mackinawite contenant du fer(III) (M*) et pyrite (P). 


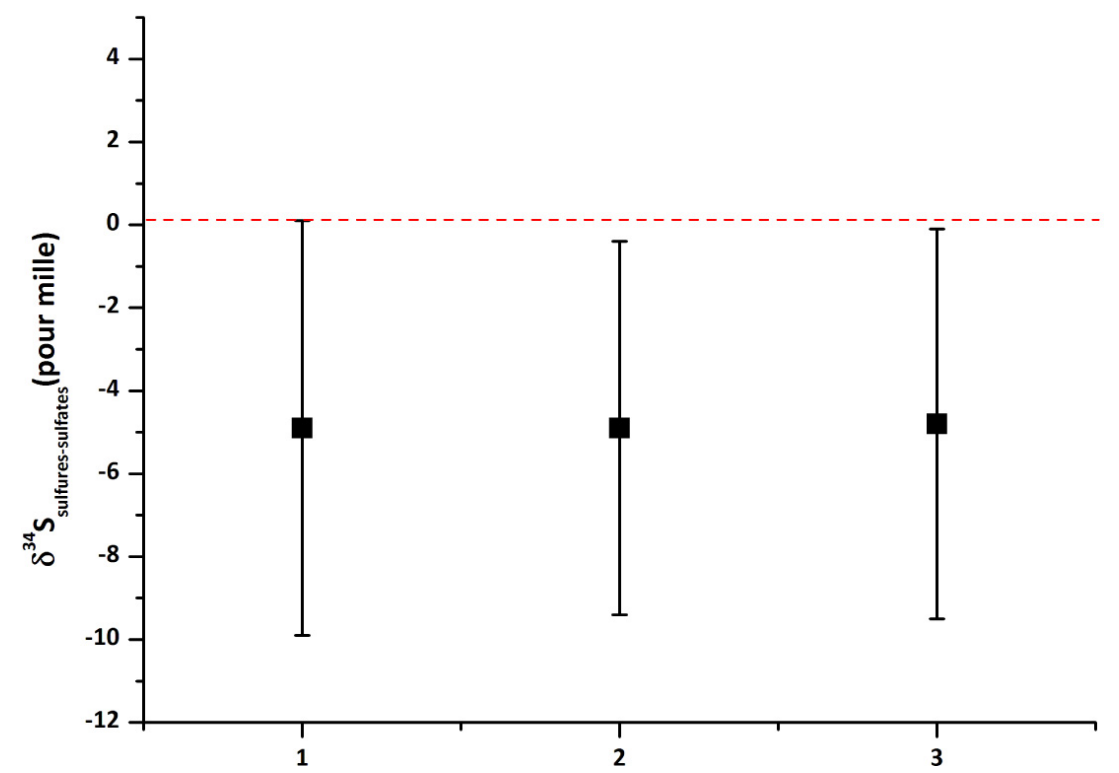

Fig. 6. Échantillon CBCC, mesures de fractionnements isotopiques des sulfures de fer par rapport aux sulfates de la solution synthétique sur les zones 1, 2 et 3 repérées sur la cartographie EDS du soufre de la Figure 4c.

\section{2 Échantillon archéologique}

En contact avec la couche de produit de corrosion se trouve une zone dorée épaisse de plusieurs centaines de micromètres. Cette zone se situe dans le milieu transformé, c'est-à-dire la zone dans laquelle sont détectés à la fois des éléments de la couche de produit de corrosion $(\mathrm{Fe}, \mathrm{C}, \mathrm{O}, \ldots)$ et des éléments des sédiments fluviaux sol (Ca, Si...). Les analyses $\mu$ Raman réalisées sur ces zones dorées résultent en un spectre unique composé de deux bandes fines et intenses à 348 et $383 \mathrm{~cm}^{-1}$ ainsi que d'une bande secondaire à $432 \mathrm{~cm}^{-1}$ caractéristiques de la pyrite (Fig. 9b).

Des analyses isotopiques ont ensuite été réalisées en nanoSIMS à la fois sur les zones greigite et/ou mackinawite de la couche de produits de corrosion (acquisitions GM10 et GM20) et sur les zones pyrite (points P5 et P6). La source initiale de sulfate ne pouvant pas être étudiée, la composition isotopique des sulfures de fer a été comparée à celle du standard international, la CDT, pour lequel ${ }^{34} \mathrm{~S} /{ }^{32} \mathrm{~S}=0,045005$. Les fractionnements isotopiques obtenus sont compris entre $-1 \pm 10 \%$ et $16 \pm 10 \%$ o (Fig. 10).

\section{Discussion}

L'échantillon CBCC a été étudié en conditions contrôlées de laboratoire en présence notamment d'une souche bactérienne sulfato-réductrice. Cet échantillon, composé d'un barreau de fer, a donc été placé en conditions corrosives dans un système dans lequel une solution synthétique composée notamment d'anions sulfate (11,2 mmol/L) circulait en continu assurant ainsi un renouvellement régulier de sulfate. Les analyses $\mu$ Raman réalisées au sein de la couche de produits de corrosion, sur les liserés dorés qui sont apparus à l'issue de l'expérience, ont permis d'identifier des sulfures de fer : la mackinawite nanocristalline, la mackinawite cristalline, la mackinawite partiellement oxydée, la greigite ainsi que de la pyrite. Ces phases se sont formées par la précipitation d'ions sulfures $\left(\mathrm{S}^{2-}\right)$ avec les ions ferreux $\left(\mathrm{Fe}^{2+}\right)$ relargués dans le milieu suite aux processus de corrosion du fer métal. Les ions sulfures du milieu sont issus dans le cas de cette expérience soit de l'activité bactérienne [13,37,38], soit de la dissolution de la pyrite contenue dans l'argilite [39-41]. En conditions anoxiques, la pyrite est peu soluble $[42,43]$ ce qui rend l'origine biotique des sulfures de fer plus probables. Et en effet, les analyses isotopiques réalisées sur les liserés de sulfures de fer et sur les poudres de sulfate commerciales ont abouti à des fractionnements isotopiques négatifs des sulfures par rapport aux sulfates de la solution porale de l'ordre de $-5 \%$. La déplétion en soufre 34 des sulfures de fer par rapport à la source initiale de sulfate confirmerait donc l'origine bactérienne des sulfures de fer observés dans cet échantillon.

Le second échantillon concerne un clou archéologique provenant du chaland gallo-romain d'Arles Rhône 3 corrodé dans des conditions non contrôlées. Dans cet échantillon, deux zones de sulfures de fer ont été distinguées : la première est située en externe de la couche de produits de corrosion et est composée d'un mélange à l'échelle submicrométrique de greigite et de mackinawite cristalline. La seconde, en revanche, est située dans le milieu transformé et est uniquement composée de pyrite.

La composition isotopique de ces sulfures de fer a été déterminée par nanoSIMS. En revanche, la composition isotopique du soufre dans la source de sulfate reste inconnue car l'échantillon s'est corrodé sur une période d'environ deux millénaires. La composition isotopique des sulfates dans l'environnement de l'échantillon a donc pu évoluer au cours de cette très longue période d'enfouis- 


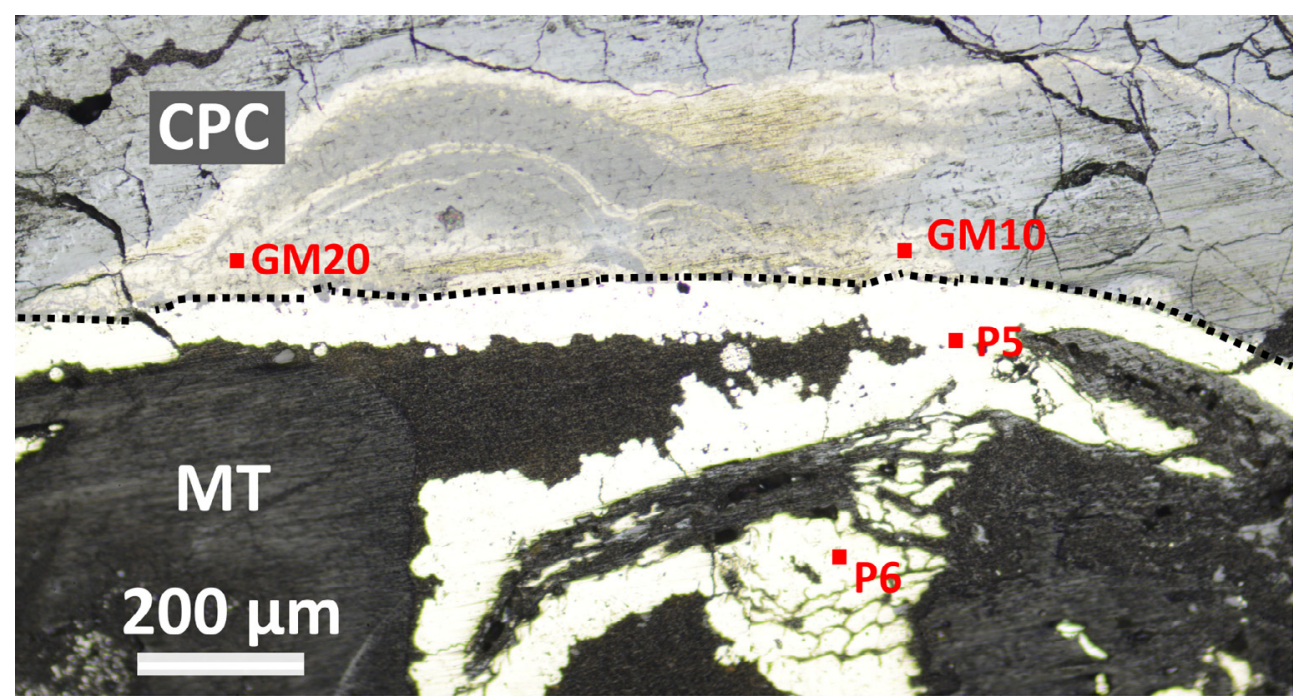

Fig. 7. Micrographie optique d'une section transverse du clou $\mathrm{T} 6 \mathrm{Fl} 2 \mathrm{Cl} 3$. Localisation de la couche de produits de corrosion (CPC) et du milieu transformé (MT).

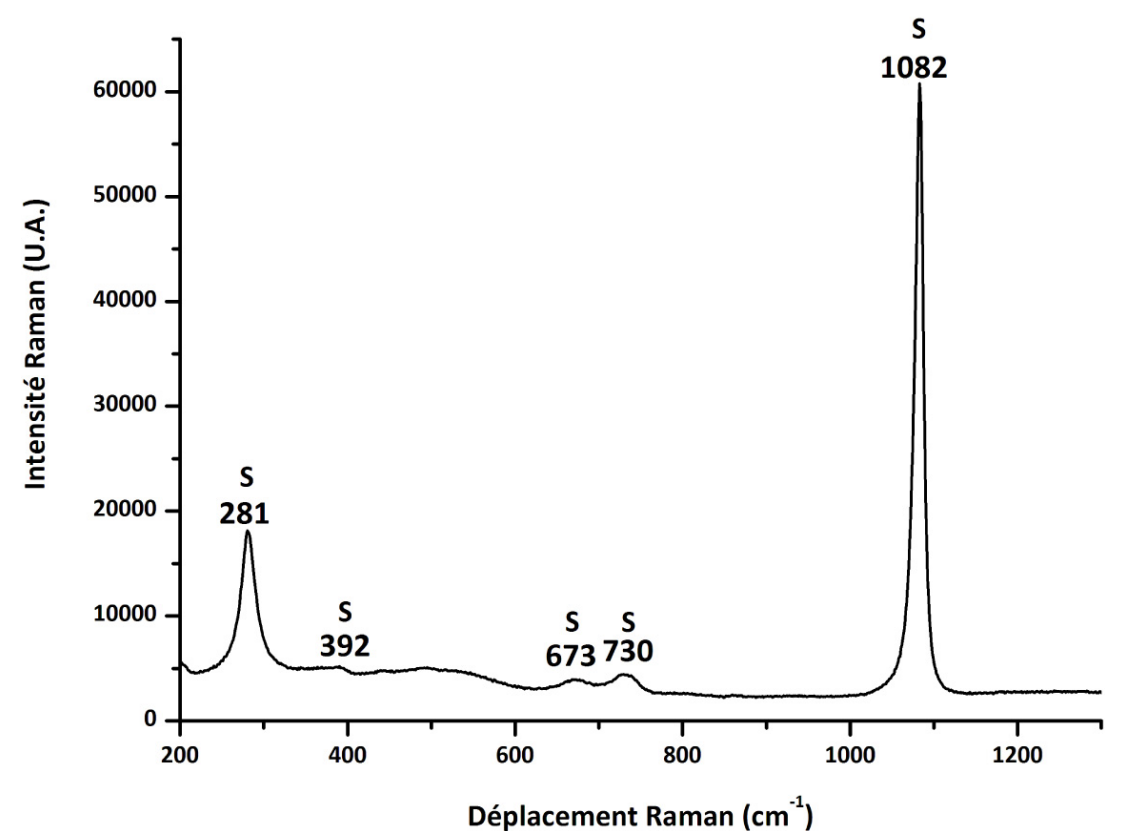

Fig. 8. Spectre $\mu$ Raman de la sidérite

sement de l'objet. Nous ne pouvons donc pas accéder à la composition isotopique de la source initiale de sulfates à proximité de l'échantillon. Les fractionnements isotopiques présentés ici ont donc été calculés par rapport à la Canon Diablo Troilite, le standard international généralement utilisé pour les études isotopiques sur le soufre $[19,44]$. Puisque l'on ne compare pas la composition isotopique des sulfures de fer de l'échantillon avec celle de la source de sulfates initiale mais seulement avec une référence arbitrairement choisie, le signe du fractionnement isotopique ne donne aucune information sur l'origine, biotique ou abiotique, des sulfures de fer observés. En effet, le fractionnement isotopique des sulfures de fer par rapport à la référence CDT peut être positif ou négatif selon que la source de sulfates est respectivement enrichie ou déplétée en soufre 34 par rapport à la CDT.

Néanmoins, malgré que la composition isotopique de la source de sulfates pendant la période de précipitation des sulfures de fer soit inconnue, il est possible d'émettre des hypothèses sur l'origine, biotique ou abiotique, des sulfures de fer dans cet échantillon en étudiant la dispersion des valeurs de fractionnements isotopiques obtenue au sein des liserés de sulfures de fer.

Nous constatons que les acquisitions réalisées dans les zones greigite/mackinawite sont comprises entre -1 $\pm 10 \%$ et $16 \pm 10 \%$. L'intervalle maximal des fractionnements isotopiques locaux dans cette zone est de l'ordre de la quarantaine de pour mille ce qui est deux fois 


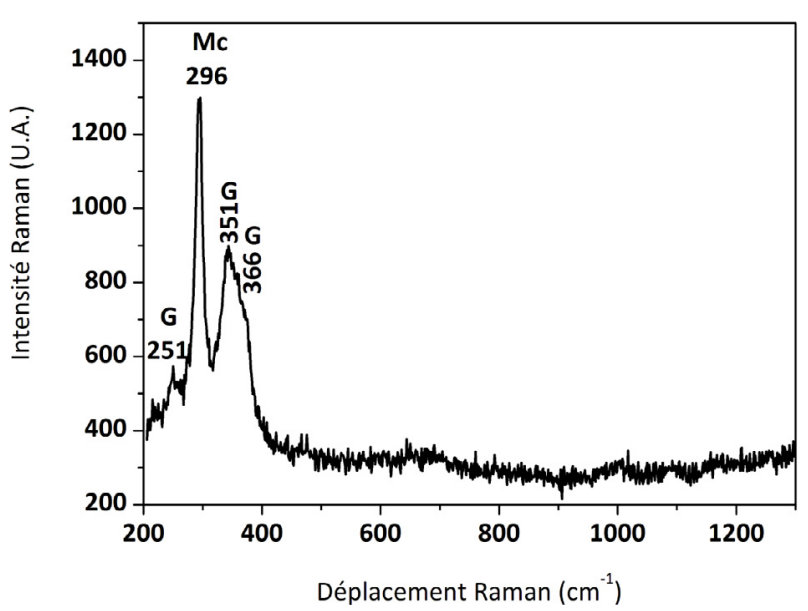

(a)

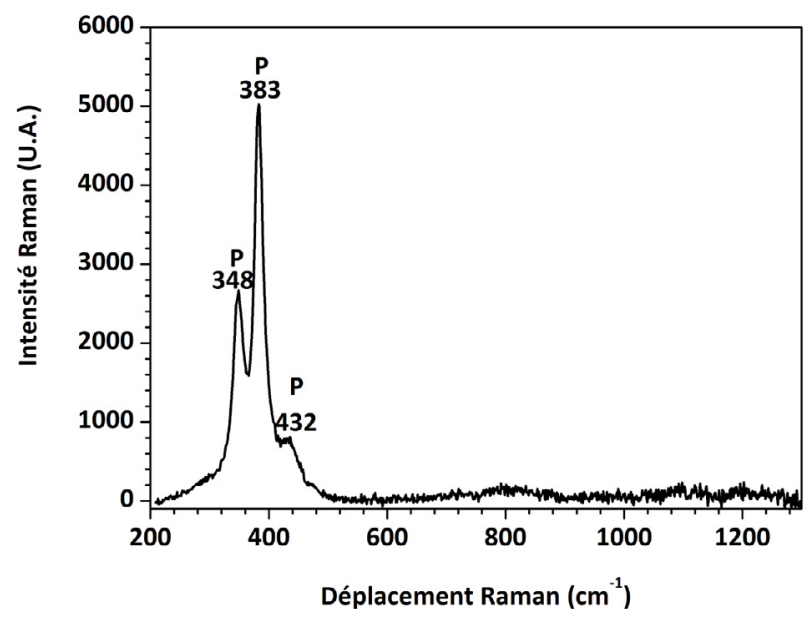

(b)

Fig. 9. Spectre Raman type des zones jaune pâle en externe de la couche de produits de corrosion (a) et des zones dorées dans le milieu transformé (b). G : Greigite; Mc : Mackinawite cristalline ; P : Pyrite.

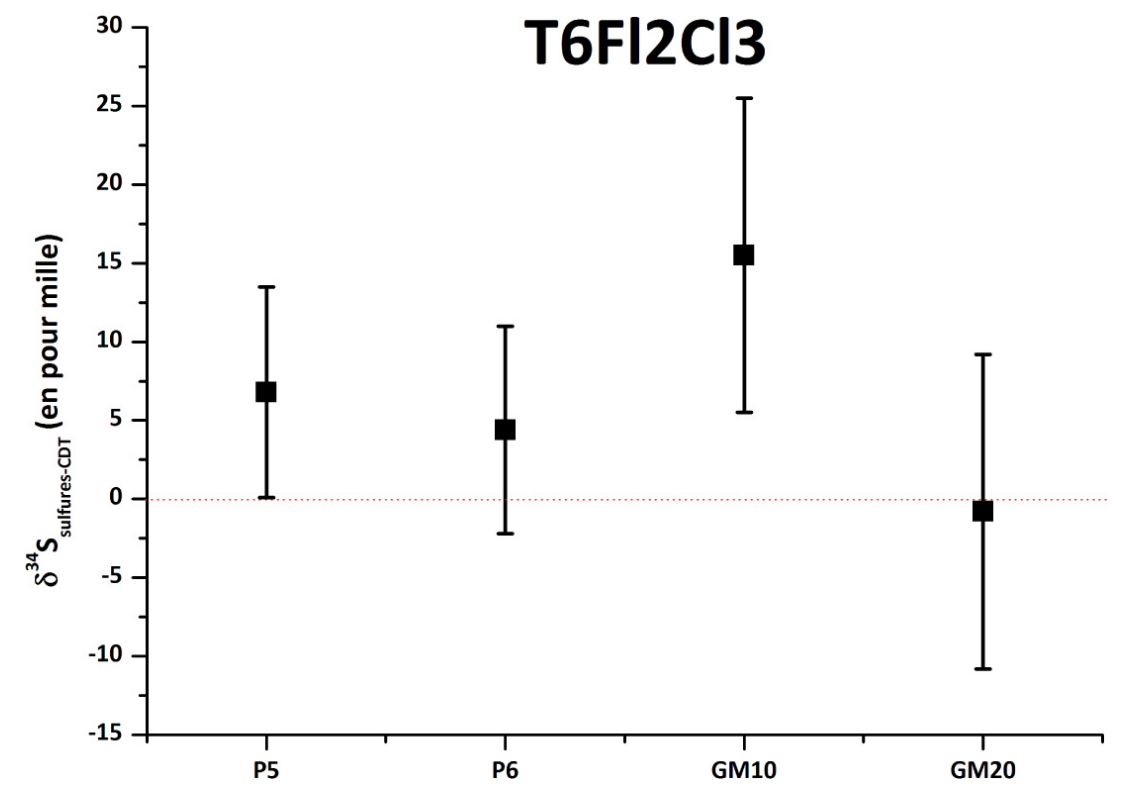

Fig. 10. Echantillon $\mathrm{T} 6 \mathrm{Fl} 2 \mathrm{Cl} 3$, fractionnements isotopiques des sulfures de fer par rapport à la référence $\mathrm{CDT}$. P5 et $\mathrm{P} 6$ : point localisés dans la zone pyrite (Fig. 7); GM10 et GM20 : points localisés dans la zone de mélange Greigite/Mackinawite (Fig. 7).

plus grand que le double de l'erreur réalisée sur la mesure du fractionnement isotopique. Si l'hétérogénéité du fractionnement isotopique au sein de ces liserés est confirmée par de nouveaux points d'analyse, deux hypothèses pourront être émises. Tout d'abord, cette dispersion pourrait résulter d'une origine bactérienne des sulfures dans un système présentant une déficience du renouvellement des sulfates. En effet, dans un premier temps, les bactéries réagissent majoritairement avec les ${ }^{32} \mathrm{SO}_{4}^{2-}$ légers mais lorsque qu'ils s'épuisent, la proportion de sulfates lourds augmente progressivement. Le rapport de consommation des ${ }^{34} \mathrm{SO}_{4}^{2-}$ et ${ }^{32} \mathrm{SO}_{4}^{2-}$ change donc cours du temps d'incubation. En début d'activité métabolique, les sulfures produits sont déplétés en soufre 34 puis peu à peu s'en- richissent en soufre 34 . Une origine bactérienne des sulfures de fer associée à un défaut de sulfates dans l'environnement immédiat pourrait donc expliquer des valeurs de fractionnements isotopiques locaux hétérogènes. Par ailleurs, puisque ces échantillons se sont corrodés pendant près de deux millénaires, l'hétérogénéité des valeurs de fractionnements isotopiques obtenue dans ces liserés de greigite/mackinawite pourrait s'expliquer par une origine biotique des sulfures de fer mais avec une composition isotopique de la source de sulfates qui évolue pendant la période de précipitation des sulfures de fer.

Les mesures du fractionnement isotopique dans les zones « pyrite » sont, quant à elles, très proches (7士 $7 \%$ et $4 \pm 7 \%$ ). La dispersion observée dans cette 
zone est faible, de l'ordre de la quinzaine de pour mille ce qui est environ égal au double de l'erreur réalisée sur la mesure du fractionnement isotopique. Si l'homogénéité $\mathrm{du}$ fractionnement isotopique au sein de ces liserés est confirmée par de nouveaux points d'analyse, deux hypothèses pourront être émises. D'une part, si les sulfates à l'origine des sulfures par mécanisme biogénique sont présents en excès dans l'environnement immédiat, la composition isotopique locale des sulfures de fer précipités au voisinage du fer métal devrait également être homogène. En effet la proportion de sulfates « légers » et « lourds » est maintenue constante du fait de l'excès de sulfates. Par conséquent, le rapport de consommation des ${ }^{34} \mathrm{SO}_{4}^{2-}$ et ${ }^{32} \mathrm{SO}_{4}^{2-}$ par les bactéries devrait être constant. Les sulfures produits devraient donc présenter une composition isotopique homogène, donc des fractionnements isotopiques locaux homogènes. D'autre part, les processus de formation non-biogéniques des sulfures de fer $[26,35,45,47-50]$ n'engendrant pas de fractionnement isotopique, les sulfures abiotiques devraient présenter des fractionnements isotopiques locaux homogènes. Il pourrait donc s'agir de phases de pyrite d'origine inorganique. Néanmoins, cet échantillon était incrusté dans le bois du chaland gallo-romain, c'est-à-dire en contact avec une grande quantité de matière organique. Sans écarter de manière certaine l'hypothèse d'une origine abiotique des liserés de pyrite, la présence d'une grande quantité de matière organique à proximité du clou rend l'hypothèse d'une origine biotique de ces liserés situés dans le milieu transformé de l'échantillon plus probable.

Ainsi, lorsque la composition isotopique de la source de sulfates est inconnue, il est intéressant d'étudier la dispersion des valeurs de fractionnements isotopiques obtenue au sein des liserés de sulfures de fer. Dans le cas d'une forte dispersion des valeurs de fractionnement isotopique, cas des liserés de mackinawite/greigite, nous pourrons conclure sur une origine bactérienne des sulfures de fer dans un système présentant un défaut de sulfates ou sur une origine bactérienne des sulfures de fer avec une composition isotopique de la source de sulfates qui évolue pendant la période de précipitation des sulfures de fer. En revanche, dans le cas d'une faible dispersion des valeurs de fractionnement isotopique, cas des liserés de pyrite, deux hypothèses restent possibles : la formation inorganique des sulfures ou bien la formation bactérienne des sulfures dans un système présentant un excès de sulfates. Seule la bonne connaissance du milieu d'enfouissement peut alors nous permettre de privilégier une hypothèse de formation par rapport à une autre. En ce qui concerne l'échantillon du site d'Arles-Rhône 3, une origine biotique des liserés de pyrite semble ainsi plus probable qu'une origine abiotique du fait de la présence de bois en grande quantité au contact de l'échantillon étudié.

\section{Conclusion}

Nous avons montré dans cette étude que l'analyse par nanoSIMS de la composition isotopique locale du soufre au sein de liserés de sulfures de fer formés au cours de processus de corrosion anoxique peut permettre de distinguer les sulfures d'origine bactérienne des sulfures d'origine inorganique. Lorsque la composition isotopique de la source de sulfates est connue, cas de l'échantillon CBCC, la détermination de l'origine des sulfures de fer est immédiate.

En revanche, pour conclure sur l'origine des sulfures de fer lorsque la composition isotopique des sulfates est inconnue, il est nécessaire de réaliser un grand nombre de points d'analyse dans des zones de sulfures de fer assez éloignées les unes des autres. Plusieurs cas sont alors possibles. Si les valeurs de fractionnements isotopiques sont très dispersées, l'hypothèse la plus probable est la formation des sulfures par les bactéries en présence d'un défaut de sulfates ou avec une composition isotopique de la source de sulfates qui évolue pendant la période de précipitation des sulfures de fer. En revanche, si les valeurs de fractionnements isotopiques sont peu dispersées, seule une bonne connaissance du milieu d'enfouissement pourra nous permettre de privilégier une origine abiotique ou une origine biotique en présence d'un excès de sulfates.

Remerciements. Nous remercions la plateforme PLATIN' (PLATeau d'Isotopie de Normandie) pour la réalisation des analyses isotopiques des poudres de sulfate de baryum utilisées dans cette étude.

\section{Annexe : Calcul du fractionnement isotopique et de son erreur à partir des analyses nanoSIMS}

Plusieurs sources d'erreur peuvent impacter la valeur de la composition isotopique obtenue en nanoSIMS par rapport à la composition isotopique réelle de l'échantillon : l'effet de l'arrivée quasi-simultanée des ions secondaires sur le détecteur ( effet QSA ») ; et le fractionnement instrumental se produisant lors du trajet des ions secondaires entre la surface de l'échantillon et le détecteur.

Tout d'abord, l'effet QSA impacte deux paramètres : le rendement d'ionisation et le fractionnement isotopique.

Le rendement d'ionisation est calculé selon la formule :

$$
K=\frac{\mathrm{Ns}}{\mathrm{Np}}
$$

avec Ns flux d'ions secondaires du fragment ionique et $\mathrm{Np}$ flux d'ions primaires total.

Dans le cas des composés soufrés, le signal du ${ }^{34} \mathrm{~S}$ est peu intense $(K<0,01)$, donc non sujet à l'effet QSA. En revanche, le signal du ${ }^{32} \mathrm{~S}$ peut être très intense $(K>$ $0,01)$. Il est donc nécessaire de corriger l'impact de l'effet QSA sur la valeur du rendement d'ionisation expérimental 
associé au soufre 32 :

$$
\begin{aligned}
K_{\exp } & =\frac{\mathrm{I} 32 \mathrm{~S}}{\mathrm{~Np}} \\
\operatorname{err}\left(K_{\exp }\right) & =\frac{\operatorname{err}(I 32 S)}{N p} \\
\text { où } \operatorname{err}(I 32 S) & =\sqrt{ }\left(\frac{N \times I 32 S}{N-I 32 S}\right)
\end{aligned}
$$

Pour cela, Slodzian et al., 2001 ont défini un rendement d'ionisation corrigé, $K_{\text {corr }}$, calculé selon la formule suivante :

$$
\begin{aligned}
K_{\mathrm{corr}} & =\frac{K_{\exp }}{1-\frac{K_{\text {exp }}}{2}}, \\
\operatorname{err}\left(K_{\mathrm{corr}}\right) & =K_{\mathrm{corr}} \times \sqrt{ }\left(\left(\frac{\operatorname{err}\left(K_{\mathrm{exp}}\right)}{K_{\exp }}\right)^{2}+\left(\frac{\operatorname{err}\left(K_{\mathrm{exp}}\right)}{1-\frac{K_{\exp }}{2}}\right)^{2}\right)
\end{aligned}
$$

En outre, une variation linéaire du fractionnement isotopique mesuré $\left(\delta_{\text {exp }}^{34}\right)$ en fonction de la valeur de $K_{\text {corr }}$ et due à l'existence de cet effet QSA, a également été mise en évidence par Slodzian et al. (2004). Afin de corriger le biais observé, l'intensité en soufre 32 est corrigée selon l'équation suivante :

$$
I 32 S_{\mathrm{corr}}=I 32 S \times\left(1+K_{\text {corr }} \times a\right),
$$

$$
\begin{aligned}
& \operatorname{err}\left(I 32 S_{\mathrm{corr}}\right)=\sqrt{ }\left(\left(1+a \times K_{\mathrm{corr}}\right)^{2} \times\left(\operatorname{err}\left(I 32 S_{\mathrm{exp}}\right)\right)^{2}\right. \\
& \left.\quad+\left(I 32 S_{\exp }\left(\frac{\operatorname{err}(a)}{a}\right)^{2}+\left(\frac{\operatorname{err}\left(K_{\mathrm{corr}}\right)}{K_{\mathrm{corr}}}\right)^{2}\right)\right)
\end{aligned}
$$

avec « $a »$ le facteur correctif de l'effet QSA correspondant à la pente de la droite $\delta_{\exp }^{34}=f\left(1000 \cdot K_{\text {corr }}\right)$.

Le rapport isotopique du soufre est alors corrigé de l'effet QSA :

$$
\begin{aligned}
R^{\prime}= & \frac{I 34 S}{I 32 S_{\mathrm{corr}}} \\
\operatorname{err}\left(R^{\prime}\right)= & R^{\prime} \times \sqrt{ }\left(\left(\frac{\operatorname{err}\left(I 32 S_{\mathrm{corr}}\right)}{I 32 S_{\mathrm{corr}}}\right)^{2}+\left(\frac{\operatorname{err}(R)}{R}\right)^{2}\right. \\
& \left.+\left(\frac{\operatorname{err}\left(I 32 S_{\mathrm{exp}}\right)}{I 32 S_{\mathrm{exp}}}\right)^{2}\right) \\
\text { où } R= & \frac{I 34 S}{I 32 S}(\mathrm{~A} .10) \\
\operatorname{err}(R)= & R \times \sqrt{ }\left(\left(\frac{\operatorname{err}(I 34 S)}{I 34 S}\right)^{2}+\left(\frac{\operatorname{err}(I 32 S)}{I 32 S}\right)^{2}\right)
\end{aligned}
$$

et $\operatorname{err}(I 34 S)=\sqrt{ }\left(\frac{N \times I 34 S}{N-I 34 S}\right)$

Par ailleurs, afin de corriger le rapport $R^{\prime}$ du fractionnement instrumental, une calibration à l'aide du standard isotopique international CDT est effectuée. Le facteur de correction $« F »$ associé est défini par l'équation :

$$
\begin{aligned}
F & =\frac{R^{\prime}(C D T)}{R(C D T) \text { theorique }} \\
\operatorname{err}(F) & =\frac{\operatorname{err}\left(R^{\prime}(C D T)\right)}{R(C D T) \text { theorique }}
\end{aligned}
$$

avec $\mathrm{R}(\mathrm{CDT})_{\text {theorique }}=0,0450045$ le rapport isotopique théorique de la CDT; et R'(CDT) le rapport isotopique expérimental de la CDT corrigé de l'effet QSA.

Le rapport $R_{\text {corr }}$ des intensités en soufre 34 et en soufre 32 corrigé à la fois de l'effet QSA et du fractionnement isotopique instrumental est alors déterminé par la formule :

$$
\begin{aligned}
R_{\text {corr }} & =\frac{R^{\prime}}{F} \\
\operatorname{err}\left(R_{\text {corr }}\right) & =R_{\text {corr }} \times \sqrt{ }\left(\left(\operatorname{err}\left(R^{\prime}\right) / R^{\prime}\right)^{2}+(\operatorname{err}(F) / F)^{2}\right)
\end{aligned}
$$

Par conséquent, le fractionnement isotopique delta ${ }^{34} \mathrm{~S}$ corrigé à la fois du QSA et du fractionnement instrumental est obtenu par la formule :

$$
\begin{aligned}
\delta^{34} S(\text { corr }) & =\frac{\left(R_{\text {corr }}-R(C D T) \text { theorique }\right)}{R(C D T) \text { theorique }} \\
\operatorname{err}\left(\delta 34 S_{\text {corr }}\right) & =1000 \times \frac{\operatorname{err}\left(R^{\prime}\right)}{R(C D T) \text { théorique }}
\end{aligned}
$$

Finalement, chaque point des graphes présentés dans cet article correspond à une unique analyse. Les barres d'erreurs associées matérialisent l'erreur finale réalisée sur le fractionnement isotopique corrigé à la fois de l'effet QSA et du fractionnement instrumental, $\delta^{34} \mathrm{~S}$ (corr).

\section{Références}

[1] V. Fell, M. Ward, Iron sulphides? : Corrosion products on artifacts from waterlogged deposits., in : Met. 98 Conf. Met. Conserv., James and James, Draguignan-Figanières, France, 1998

[2] Y. Fors, F. Jalilehvand, E. Damian Risberg, C. Björdal, E. Phillips, M. Sandström, J. Archaeol. Sci. 39 (2012) 2521-2532

[3] Y. Fors, T. Nilsson, E.D. Risberg, M. Sandström, P. Torssander, Int. Biodeterior. Biodegrad. 62 (2008) 336347

[4] V.S. Sastri, 5 - Corrosion processes and the use of corrosion inhibitors in managing corrosion in underground pipelines A2 - Orazem, Mark E., in : Undergr. Pipeline Corros., Woodhead Publishing, 2014 : pp. 127-165. http://www. sciencedirect.com/science/ article/pii/B9780857095091500059

[5] B. James, A. Hudgins, Chapter 1 - Failure analysis of oil and gas transmission pipelines A2 Aliofkhazraei, Abdel Salam Hamdy MakhloufMahmood, in : Handb. Mater. Fail. Anal. Case Stud. Oil Gas Ind., Butterworth-Heinemann, 2016 : pp. 138. http://www.sciencedirect.com/science/article/ pii/B9780081001172000017 
[6] C. Chautard, J.-E. Lartigue, M. Libert, F. Marsal, L. De Windt, Procedia Chem. 7 (2012) 641-646

[7] M. Saheb, D. Neff, P. Dillmann, H. Matthiesen, E. Foy, L. Bellot-Gurlet, Mater. Corros. 60 (2009) 99-105

[8] R.A. King, J.D.A. Miller, Br. Corros. J. 8 (1973)

[9] J. Crolet, Mater. Tech. 80 (1992) 71-77

[10] R. Marchal, Oil Gas Sci. Technol. 54 (1999) 649-659

[11] W. Sun, S. Nešić, Corrosion 65 (2009) 291-307

[12] Y. Zheng, B. Brown, S. Nešić, Corrosion 70 (2013) 351365

[13] C. Lemaître, N. Pebere, D. Festy, Biodétérioration des matériaux, EDP Sciences, 1998

[14] D.E. Canfield, Geochim. Cosmochim. Acta 65 (2001) $1117-1124$

[15] M.S. Sim, T. Bosak, S. Ono, Science 333 (2011) 74-77 DOI : $10.1126 /$ science. 1205103

[16] G. Antler, A.V. Turchyn, V. Rennie, B. Herut, O. Sivan, Geochim. Cosmochim. Acta 118 (2013) 98-117

[17] M.C. Stam, Sulfur isotopes as a tracer for biogenic sulfate reduction in natural environments : A link between modern and ancient ecosystems, 2010

[18] C. Kendall, E.A. Caldwell, Fundamentals of Isotope Geochemistry, in : Isot. Tracers Catchment Hydrol., C. Kendall and J.J. McDonnell, Amsterdam, 1998, pp. 5186

[19] H.G. Thode, J. Monster, H.B. Dunford, Geochim. Cosmochim. Acta 25 (1961) 159-174

[20] Y. Kajiwara, H.R. Krouse, A. Sasaki, Earth Planet. Sci. Lett. 7 (1969) 271-277

[21] B. Bühn, R.V. Santos, M.A. Dardenne, C.G. de Oliveira, Chem. Geol. 312-313 (2012) 163-176

[22] P. Aharon, B. Fu, Geochim. Cosmochim. Acta 64 (2000) 233-246

[23] D.E. Canfield, C.A. Olesen, R.P. Cox, Geochim. Cosmochim. Acta $\mathbf{7 0}$ (2006) 548-561

[24] J. Kleikemper, M.H. Schroth, S.M. Bernasconi, B. Brunner, J. Zeyer, Geochim. Cosmochim. Acta 68 (2004) 4891-4904

[25] T. Ding, S. Valkiers, H. Kipphardt, P. De Bièvre, P.D.P. Taylor, R. Gonfiantini, R. Krouse, Geochim. Cosmochim. Acta 65 (2001) 2433-2437

[26] C. Chautard, Intéractions fer/argile en conditions de stockage géologique profond - Impacts d'activités bactériennes et d'hétérogénéités, Thèse, École nationale supérieure des mines de Paris, 2013

[27] J. Tremosa, D. Arcos, J.M. Matray, F. Bensenouci, E.C. Gaucher, C. Tournassat, J. Hadi, 25 Years Chernobyl Power Plant Explos. Manag. Nucl. Wastes Radionucl. Transf. Environ. 27 (2012) 1417-1431
[28] C. Beaucaire, J.-L. Michelot, S. Savoye, J. Cabrera, Appl. Geochem. 23 (2008) 2182-2197

[29] O. Haouari, M.-L. Fardeau, J.-L. Cayol, G. Fauque, C. Casiot, F. Elbaz-Poulichet, M. Hamdi, B. Ollivier, Syst. Appl. Microbiol. 31 (2008) 38-42

[30] C. Jeanthon, A.-L. Reysenbach, S. L'Haridon, A. Gambacorta, N.R. Pace, P. Glénat, D. Prieur, Arch. Microbiol. 164 (1995) 91-97

[31] J. Kostka, K.H. Nealson, Tech. Microb. Ecol. (1998) 5878

[32] S. Marlier, Arles-Rhône 3 : un chaland gallo-romain du Ier siècle aprèsJésus-Christ, Éd. du CNRS, Paris, 2014

[33] A. Boughriet, R.S. Figueiredo, J. Laureyns, P. Recourt, J. Chem. Soc. Faraday Trans. 93 (1997) 3209-3215

[34] J.-A. Bourdoiseau, M. Jeannin, R. Sabot, C. Rémazeilles, P. Refait, Corros. Sci. 50 (2008) 3247-3255

[35] J.-A. Bourdoiseau, M. Jeannin, C. Rémazeilles, R. Sabot, P. Refait, J. Raman Spectrosc. 42 (2011) 496-504

[36] S.N. White, Chem. Geol. 259 (2009) 240-252

[37] H.G. Machel, Sediment. Geol. 140 (2001) 143-175

[38] C. Harerimana, B. Harbi, J.-L. Vasel, Agron. Société Environ. 14 (2010) 577-582

[39] A. Gaudin, S. Gaboreau, E. Tinseau, D. Bartier, S. Petit, O. Grauby, F. Foct, D. Beaufort, Appl. Clay Sci. 43 (2009) 196-207

[40] D. Charpentier, M. Cathelineau, R. Mosser-Ruck, G. Bruno, Comptes Rendus Académie Sci. Sér. 2 Sci. Terre Planètes 332 (2001) 601-607

[41] M. Descostes, Evaluation d'une perturbation oxydante en milieu argileux? : mécanisme d'oxydation de la pyrite $\left(\mathrm{FeS}_{2}\right)$, Thèse, Université Paris VII, 2001

[42] R.A. Berner, Am. J. Sci. 268 (1970) 1-23

[43] R.A. Berner, Cosmochim. Acta 48 (1984) 605-615

[44] G. Beaudoin, B.E. Taylor, D. Rumble III, M. Thiemens, Geochim. Cosmochim. Acta 58 (1994) 4253-4255

[45] R.T. Wilkin, H.L. Barnes, Geochim. Cosmochim. Acta 61 (1997) 323-339

[46] I.B. Butler, D. Rickard, Geochim. Cosmochim. Acta 64 (2000) 2665-2672

[47] A.G. Wikjord, T.E. Rummery, F.E. Doern, D.G. Owen, Corros. Sci. 20 (1980) 651-671

[48] S. Hunger, R.J. Newton, S. Bottrell, L.G. Benning, Geochim. Cosmochim. Acta 70 (2006) A273

[49] S. Hunger, L.G. Benning, Geochem. Trans. 8 (2007) 1-20

[50] D. Rickard, G.W. Luther, Chem. Rev. 107 (2007) 514562 